\title{
Setting standards for physical health monitoring in patients on antipsychotics
}

\section{AIMS AND METHOD}

To develop standards for physical health monitoring in patients on antipsychotics in a forensic environment. We reviewed recommendations for physical health checks from key publications and then agreed a consensus on what to monitor and how often.

\section{RESULTS}

We developed a standardised form to ensure the requisite minimum monitoring is performed when a patient is newly prescribed an antipsychotic drug or the dose is increased, when an abnormality is detected and for routine annual physical health monitoring.

\section{CLINICAL IMPLICATIONS}

We have produced a pragmatic solution to the gap between the recognised risks to physical health of individuals treated with antipsychotic medication and the need for clear, unambiguous standards of physical healthcare.
There is now extensive evidence that people with severe mental illness have higher rates of morbidity and mortality for common physical illnesses than the general population. ${ }^{1}$ However, in forensic settings there is a dearth of data on morbidity rates, but the limited evidence available does suggest significant rates of physical health problems for in-patients in secure settings. 2,3

A number of publications have highlighted the need for physical health monitoring in individuals with mental health problems. ${ }^{4}$ Despite that, there is no established national consensus detailing precisely what should be measured and how frequently.

We are based in a forensic mental health service without any general practitioner input; patients can be resident for periods beyond 10 years. Primary care services are provided by psychiatric trainees and staff grade doctors.

The National Institute for Health and Clinical Excellence (NICE) guidelines on schizophrenia refer to primary and secondary services with regard to the provision of care to individuals with schizophrenia. ${ }^{5}$ Criterion 12 of the guidelines highlights a minimum standard of physical health checks 'at regular intervals' for those with schizophrenia within primary care. We carried out an audit of this criterion within our in-patient service that demonstrated deficits in the measurement and recording of physical health parameters. ${ }^{6}$ The need for a more systematic approach to the execution and documentation of physical health checks has been highlighted by our audit and by other services. ${ }^{7}$ This paper addresses this need.

There is increasing evidence of the link between antipsychotic medication and physical health risks, such as:

- weight gain 8

- metabolic syndrome9,10

- endocrine problems (e.g. diabetes, 11,12 hyperprolactinaemia, ${ }^{13,14}$ sexual dysfunction ${ }^{15}$ )

- cardiovascular problems

- sudden death ${ }^{16,17}$
- abnormalities of QTC ${ }^{18}$

- effects on blood pressure ${ }^{19}$

- stroke. $^{20}$

In the light of a high percentage (87\%) of our in-patients being prescribed antipsychotic medication (Churchward et al, 2007 internal audit, Langdon Hospital, personal communication) and the potentially elevated risks in this group, we decided to set standards for physical health checks that would incorporate the risks associated with the institution and ongoing treatment with antipsychotics.

\section{Method}

A multidisciplinary group was formed in June 2006, comprising a senior mental health pharmacist (S.C.) and three staff psychiatrists (D.T., V.O., S.O.).

A widespread search of the English language publications to date was carried out to establish the relevant evidence base. The relevant papers were then reviewed and the key points summarised with respect to the physical health parameters that had been identified as important. A pragmatic consensus was agreed on by the group with regard to the parameters of what should be measured and how often.

Once standards had been agreed, other colleagues on site, from a variety of disciplines, were consulted to determine the most appropriate format for the implementation of the standards. We explored the current development of the NHS Care Records Service (NCRS), particularly the electronic patient records service, both locally and nationally. ${ }^{21}$ Although the NCRS has the potential to include monitoring, we found that in its current state of development it does not have the flexibility to incorporate this type of tailor made monitoring. ${ }^{22}$

An example of the summary of the key points from the review is presented in Table 1 (full review of recommendations on all parameters available from the authors on request). 


\section{Results}

Having collated the data, it was evident that there was a original papers

lack of uniformity from the different sources on how often the parameters should be measured (Table 1). A major obstacle to improving the quality of physical healthcare monitoring for patients with schizophrenia is the lack of consensus regarding which health parameters should be monitored and when they should be monitored. ${ }^{4}$

We recognise the importance of good primary care services for patients in forensic mental health services. However, this project did not attempt to incorporate all aspects of preventative healthcare but focused specifically on monitoring elements of physical health risk compounded by treatment with antipsychotic medication.

\section{Parameters measured}

We reached a consensus on which parameters should be measured and how often, based on the evidence we collated (Table 2). Where the evidence from the literature suggested monitoring less frequently than our own clinical experience, we agreed a frequency that we felt was clinically appropriate for our patient group. In particular, the recommendations on how often to weigh patients were rather infrequent compared with our current clinical practice. Given the ease of monitoring this parameter and the importance of this risk factor for our patients, we reached a consensus to continue to monitor weight on a monthly basis.

Recommendations on some parameters such as electrocardiogram (ECG) varied considerably in the literature, ranging from monitoring ECG at baseline, ${ }^{18}$ after each dose escalation and 6-monthly monitoring, ${ }^{29}$ to monitoring 6-monthly for higher risk drugs. ${ }^{25} \mathrm{We}$ reached a consensus taking into consideration the recommendations available and our own clinical judgement. We already had a procedure in place for the monitoring of patients prescribed high-dose antipsychotics, based on the Royal College of Psychiatrists guidelines. ${ }^{30}$ Patients prescribed above the British National Formulary maximum dosages of antipsychotics 24 are routinely monitored with an ECG every 3 months. A consensus was reached to do a baseline ECG for all patients on admission and to do ECG monitoring every 3 months for patients prescribed high-dose antipsychotics or those on higher risk antipsychotics (pimozide, sertindole). We acknowledged that there were other circumstances where repeat ECG may be necessary, such as in patients with cardiovascular disease or following rapid tranquillisation, but felt that this was beyond the scope of our standards and that such cases would need appropriate individual care planning.

Regarding hyperprolactinaemia, the literature does not indicate a consensus on routine or even baseline monitoring of prolactin levels. Regular monitoring for signs of prolactin elevation or sexual dysfunction is, however, recommended. ${ }^{4,31}$ The clinical consequences of hyperprolactinaemia include menstrual disturbance, galactorrhoea and sexual dysfunction. These clinical features are incorporated within the Liverpool University Neuroleptic Side Effect Rating Scale (LUNSERS). ${ }^{32}$

Some side-effects of antipsychotics may be rare but are still significant to the patient such as the risk of cataract with phenothiazines and quetiapine. ${ }^{4}$ We

Table 1. Comparison of recommendations for physical health monitoring in patients treated with antipsychotic medication (example)

Summary

of APBI Medi-

cines Com-

pendium ${ }^{23}$ or $B N F^{24}$

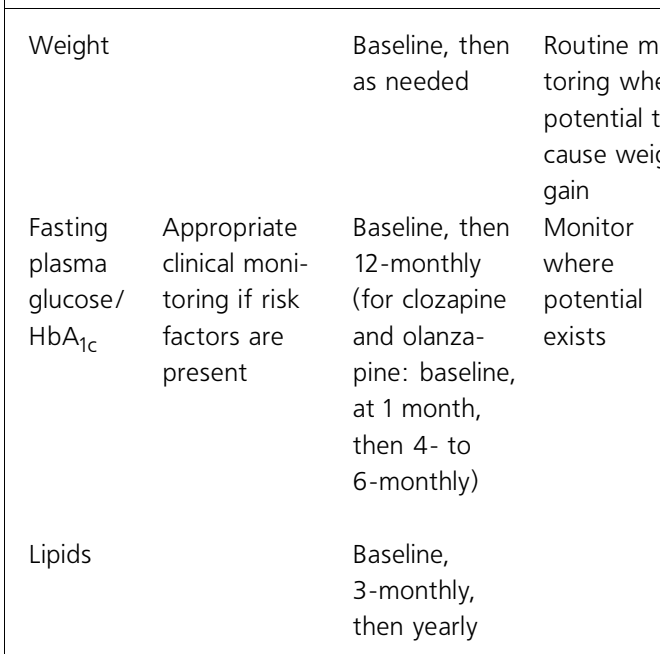

NICE

schizophrenia

guidelines ${ }^{5}$

Lebovitz $^{26}$

Baseline, then periodically

\section{Baser}

Baseline, then

months

Baseline, at 6 weeks, then every 3 months

Baseline, at 6 weeks, at 6 months, then yearly
Antai-Otong 27

Marder et $a l^{4}$

American Diabetes Association et $a^{28}$

Baseline, then Baseline, at monthly for first 6 months, then every quarter once dose stabilised

4 weeks, at 8 weeks, then Baseline, then Baseline; if signifiyearly (moni- cant risk factors for tor for signs diabetes ${ }^{\mathrm{a}}$ are 3-monthly Baseline, at 3 months and then of weight present, measure at yearly gain, polyuria, baseline, at 4 months polydypsia) and then annually. For patients gaining weight, measure every 4 months

Baseline, then Baseline, then every every 2 years 2 years; if LDL $>130 \mathrm{mg} / \mathrm{dl}$ then Baseline, at 3 months, then every every 6 months 5 years

BNF, British National Formulary; $\mathrm{HbA}_{1 \mathrm{c}}$ glycosylated haemoglobin; LDL, low-density lipoprotein.

a. Family history, body mass index $\geqslant 25$, waist circumference $\geqslant 35$ inches for women and $\geqslant 40$ inches for men. 
Table 2. Monitoring schedule required for all patients on antipsychotic medication $^{\mathrm{a}}$

\begin{tabular}{|ll}
\hline When & Parameters \\
\hline On admission & Height \\
& Weight \\
& Waist circumference \\
& Glucose \\
& Lipids \\
& Electrocardiogram \\
& Blood pressure \\
& Liver function test \\
& Urea and electrolytes \\
& Thyroid function test \\
& Full blood count \\
& LUNSERS \\
& Weight \\
& Smoking \\
& Waist circumference \\
Monthly & Glucose \\
& Lipids \\
Annually & Electrocardiogram ${ }^{2}$ \\
& Blood pressure \\
& Liver function test \\
& Urea and electrolytes \\
& Thyroid function test \\
& Full blood count \\
& Eye examination $(>40$ years old) \\
& LUNSERS \\
& Eye examination ( $>40$ years old) \\
&
\end{tabular}

LUNSERS, Liverpool University Neuroleptic Side Effect Rating Scale.

a. If any abnormalities are detected or the patient has particular medical risk factors, monitoring will be required as appropriate for the individual patient.

b. Electrocardiogram monitoring should be repeated 3-monthly for patients

on high-dose antipsychotics or higher risk drugs such as thioridazine, sertindole

and pimozide.

therefore agreed to incorporate regular eye checks in our local standard for physical health checks in patients on antipsychotics.

We decided to include smoking in our final monitoring form as it is such an important lifestyle factor known to have impact on the risks that we are addressing in this paper.

We were keen to transform Table 2 into a format that would be more practical at the ward level and serve to prompt clinicians as to when monitoring was due. Consequently, we developed the physical health monitoring form (see online supplement to this paper).

Two other forms were devised (available from the authors on request) for annual monitoring of haematological and biochemical parameters as well as further monitoring of any abnormal result findings, but for the purpose of this paper we limited ourselves to the first year of antipsychotic administration.

\section{Implementation}

Our results were presented to the forensic multidisciplinary clinical governance meeting. Agreement was reached to incorporate the standards in a policy and procedure. Each patient would have a physical health form filed in a separate physical health folder, which could be checked at every clinical review meeting. Nursing staff and medical staff shared responsibility for collecting and recording data.

\section{Discussion}

The importance of monitoring physical health in all patients with mental health problems is apparent from current evidence. In population terms, the impact of treatment with antipsychotic medication is potentially compounding an already elevated risk of cardiovascular and metabolic disease in this patient group.

The lack of clear guidelines on how to implement NICE guidance is well known. ${ }^{33}$ We took a pragmatic approach using a consensus agreement between colleagues to create standards that we then implemented through the creation of structured monitoring forms, which can be translated into a computer-based system when available.

We hope that others may find this a useful model that could be applicable with modification in other settings.

Future areas of work could include development of algorithms for the management of abnormal findings (e.g. elevated blood sugar, hyperlipidaemia), exploration of patients' perspective on monitoring of physical health, and re-auditing of completed physical health monitoring using the new standards and recording sheets.

We would like to see clear standards for physical healthcare and a structured monitoring system included in the NCRS.

\section{Declaration of interest}

None.

\section{References}

1 Cohen A, Hove M. Physical Health of the Severe and Enduring Mentally III. Sainsbury Centre for Mental Health, 2001

2 Sebastian C, Beer MD. Physica health of psychiatric patients admitted to a low secure challenging behaviour unit. J Psychiatr Intensive Care 2006; 1: 77-83.

3 Thomson L, Bogue J, Humphreys $\mathrm{M}$ Owens D, Johnstone E. The State Hospital Survey: a description of psychiatric patients in conditions of special security in Scotland. J Forensic Psychiatr 1997; 8 263-84.

4 Marder SR, Essock SM, MillerAL, Buchanan RW, Casey DE, Davis JM, et al. Physical health monitoring of patients with schizophrenia. Am J Psychiatry 2004; 161: 1334 -49.

5 National Institute for Clinical Excellence. Schizophrenia: Core
Interventions in the Treatment and Management of Schizophrenia in Primary and Secondary Care. NICE, 2002.

6 Gough K, Churchward S, Dorkins E, Fee J, Oxborrow S, Parker J, et al. Audit of the NICE Guidelines for Schizophrenia in an NHS forensic psychiatric service. BrJ Forensic Pract 2007; 9: 28-34.

7 Singh R, Ahmed MJR, Shaffiullha M. Audit of monitoring of second generation antipsychotic drugs. Interest Group of Royal College of Psychiatrists, Autumn 2007,10-6.

8 Zhang Z-J, Yao Z-J, LiuW, Fang Q, Reynolds GP. Effects of antipsychotics on fat deposition and changes in leptin and Insulin levels. Magnetic resonance imaging study of previously untreated Psychiatry 2004; 184: 58-62. Bulletin of Transcultural Special people with schizophrenia. $\mathrm{Br}$ J 
9 McCreadie RG. Diet, smoking and cardiovascular risk in people with schizophrenia. Descriptive study. $\mathrm{Br}$

original papers

J Psychiatry 2003; 183: 534 -9.
10 Thakore JH. Metabolic syndrome and schizophrenia. BrJ Psychiatry 2005: 186: 455-6.

11 Haddad PM. Antipsychotics and diabetes: review of nonprospective data. BrJ Psychiatry 2004; 184 (suppl 47): s80-6.

12 LambertT, Chapman L. Diabetes, psychotic disorders and antipsychotic therapy: a consensus statement. Med J Aust 2004; 181: 544-8.

13 Petty RG. Prolactin and antipsychotic medications: mechanism of action. Schizophr Res 1999; 35 (suppl 1): 67-73.

14 Holt RIG. The medical causes and consequences of

hyperprolactinaemia. A context for psychiatrists. J Psychopharmacol 2008; 22 (suppl 2): 28-37.

15 Berner MM, Hagen M, Kriston L. Management of sexual dysfunction due to antipsychotic drug therapy. Cochrane Database Syst Rev 2007; Issue 3: CD003546.

16 Herxheimer A, Healy D. Arrhythmias and sudden death in patients taking antipsychotic drugs. BMJ 2002; 325: 1253-4.

17 RayWA, Meador KG.

Antipsychotics and sudden death: is thioridazine the only bad actor? BrJ Psychiatry 2002; 180

483-4.

18 Abdelmawla N, Mitchell AJ. Sudden cardiac death and antipsychotics. Part 2: Monitoring and prevention. Adv Psychiatr Treat 2006; 12 $100-9$.

19 Haddad PM, Sharma SG. Adverse effects of atypical antipsychotics: differential risk and clinical implications. CNS Drugs 2007; 21 911-36.

20 Gill SS, Rochon PA, Herrmann N, Philip EL, Sykora K, Gunraj N, et al. Atypical antipsychotic drugs and risk of ischaemic stroke: population based retrospective cohort study. BMI2005: 330: 445

21 NHS Connecting for Health. NHS Care Record Service. NHS, 2005.

22 Brennan S. The NHS IT Project: The Biggest Computer Project in the World Ever. Radcliffe Publishing, 2005.

23 Association of the British Pharmaceutical Industry (ABPI): ABPI Medicines Compendium.
Datapharm Communications, 2006 (http://www.emc.medicines. org.uk).

24 British Medical Association, Roya Pharmaceutical Society of Great Britain. British National Formulary 51. BMA, 2006

25 David T, Paton C, Kerwin R. The Maudsley 2005-2006 Prescribing Guidelines (8th edn). Taylor and Francis, 2005.

26 Lebovitz HE. Metabolic consequences of atypical antipsychotic drugs. Psychiatr $Q$ 2003; 74: 277-90.

27 Antai-Otong D. Metabolic effects associated with atypical antipsychotic medications. Perspect Psychiatr Care 2004; 40 $70-2$.

28 American Diabetes Association, American Psychiatric Association, American Association of Clinical Endocrinologists, North American Association for the Study of Obesity. Consensus development conference on antipsychotic drugs and obesity and diabetes. Diabetes Care 2004: 27: 596-601

29 Medicines Control Agency, Committee on Safety of Medicines. QT interval prolongation with antipsychotics. Curr Probl Pharmacovigilance 2001; 27: 4

30 Royal College of Psychiatrists. Consensus Statement on HighDoseAntipsychotic Medication (R138). Royal College of Psychiatrists, 2006

31 Maguire GA. Prolactin elevation with antipsychotic medications: mechanisms of action and clinical consequences. J Clin Psychiatry 2002; 63 (suppl 4): 56-62.

32 DayJC,Wood G, Dewey M, Bentall RP. A self-rating scale for measuring neuroleptic side-effects. Validation in a group of schizophrenic patients. BrJ Psychiatry 1995; 166 $650-3$.

33 Rowlands P. The NICE schizophrenia guidelines: the challenge of implementation. Adv PsychiatrTreat 2004; 10: 403-12

Samantha Churchward Forensic Services Pharmacist, Devon Partnership NHS Trust, Langdon Hospital, Devon, Susan M. Oxborrow Staff Grade Psychiatrist, Bluebird House, Southampton, *Victor O. Olotu Staff Grade Psychiatrist, Langdon Hospital, Exeter Road, Dawlish, Devon, email: victor.olotu @nhs.uk, M. DeepakThalitaya Specialist Trainee Registrar, Brooklands Hospital, Brian Oliver Centre, Birmingham

\section{Survey of Scottish psychiatrists' views on neuropsychology training}

\author{
AIMS AND METHOD \\ A national survey of members of \\ the Scottish division of the Royal \\ College of Psychiatrists to \\ establish the training in \\ neuropsychology they have \\ received, their views on \\ neuropsychology service provision \\ and on potential formal \\ postgraduate neuropsychology \\ training opportunities.
}

\author{
RESULTS \\ A $54 \%(n=288)$ consultant \\ psychiatrist response rate was \\ achieved. The responses suggest that \\ where specialist neuropsychology \\ services exist, they are not perceived \\ to be sufficient to deal with clinical \\ demand. Consultant psychiatrists \\ indicated that there are limited \\ training opportunities in neuro- \\ psychology for psychiatrists, and this \\ seems to be reflected in their limited
}

confidence in interpreting basic recommended neuropsychological assessment tools. There seems to be a definite desire among consultant psychiatrists for postgraduate training opportunities.

\section{CLINICAL IMPLICATIONS}

Scottish psychiatrists desire an increase in formal neuropsychology training opportunities.
The application of neuropsychology is relevant to the majority of psychiatric subspecialties. This is affirmed in various national clinical guidelines: old age psychiatry in relation to the neurodegenerative disorders ${ }^{1-3}$ as well as psychiatry of learning disability and child and adolescent psychiatry in relation to the neurodevelopmental conditions that are clinically eloquent early in life. ${ }^{4-8}$ Specialists in general adult psychiatry and forensic psychiatry may encounter individuals affected with the aforementioned conditions. ${ }^{9}$ They also treat people with severe and enduring mental illness with neuropsychological sequelae, particularly the chronic psychotic disorders. It is important to distinguish the latter from other neurodegenerative condition, especially in senescence. ${ }^{10}$ The neuropsychological assessment of those with suspected alcohol-related brain damage is 